\title{
Radial variation of Bloch functions on the unit ball of $\mathbb{R}^{d}$
}

\author{
Paul F. X. Müller and Katharina Riegler
}

\begin{abstract}
In [9] Anderson's conjecture was proven by comparing values of Bloch functions with the variation of the function. We extend that result on Bloch functions from two to arbitrary dimension and prove that

$$
\int_{[0, x]}|\nabla b(\zeta)| e^{b(\zeta)} d|\zeta|<\infty .
$$

In the second part of the paper, we show that the area or volume integral

$$
\int_{B^{d}}|\nabla u(w)| p(w, \theta) d A(w)
$$

for positive harmonic functions $u$ is bounded by the value $c u(0)$ for at least one $\theta$. The integral is also transferred to simply connected domains and interpreted from the point of view of stochastics. Several emerging open problems are presented.
\end{abstract}

\section{Introduction}

In [9] it was proven that for every Bloch function $b$ on the unit disc there is a point $x$ on the unit circle such that

$$
\int_{[0, x]}|\nabla b(\zeta)| e^{b(\zeta)} d|\zeta|<\infty .
$$

This fact was used in [9] to show Anderson's conjecture for conformal maps on the unit disc. The proof used the result of Bourgain ([2]) that for every positive harmonic function there is a direction of bounded radial variation, Pommerenke's

Key words and phrases: radial variation, Bloch functions.

2010 Mathematics Subject Classification: 31B25, 30H30, 31A20. 
theorem on the existence of a dense set of rays along which a Bloch function on the unit disc remains bounded (see [15, Proposition 4.6.]) and conformal mappings between starlike Lipschitz domains and the unit disc. The result of Bourgain ([2]) was extended to half-spaces by Michael O'Neill in [14] and even to higher dimensional Lipschitz domains by Mozolyako and Havin in [11]. The extension to Lipschitz domains in higher dimensions by Mozolyako and Havin was the starting point of our work in this paper. The main result of the present paper is the following Theorem 1, extending the result of [9] to the unit ball of $\mathbb{R}^{d}$. We refer to [13] and [14] where this problem was posed in writing.

Theorem 1. Let $b$ be a Bloch function on the unit ball $B$ of $\mathbb{R}^{d}$. Then there is a point $x$ on the unit sphere such that

$$
\int_{[0, x]}|\nabla b(\zeta)| e^{b(\zeta)} d|\zeta|<\infty .
$$

Remarks The proof of Theorem 1 will show as well that there is an $x$ such that for $y \in[0, x]$ and $1-|y|$ small enough we have

$$
b(y) \leq b(0)-c \int_{[0, y]}|\nabla b(\zeta)| d|\zeta| .
$$

As $-b$ is also a Bloch function we get a point $\tilde{x}$ on the unit sphere such that for $y \in[0, \tilde{x}]$ and $1-|y|$ small enough we have

$$
b(y) \geq b(0)+c \int_{[0, y]}|\nabla b(\zeta)| d|\zeta| .
$$

This can also be written as

$$
\liminf _{y \rightarrow 1} \frac{b(y)-b(0)}{\int_{[0, y]}|\nabla b(\zeta)| d|\zeta|}>0 .
$$

Comparing our proof of Theorem 1 with the one in [9] we observe the following.

1. The use of conformal maps onto Lipschitz domains is replaced by the result of Mozolyako and Havin.

2. In [9] the straight line segments obtained by Pommerenke's theorem are used to suitably cut planar domains. While Pommerenke's result on the radial behaviour of Bloch functions is still valid in $\mathbb{R}^{d}$ for $d>2$ (see Nicolau [13]) the corresponding line segments cannot be used to separate domains in $\mathbb{R}^{d}$ for $d>2$ (note that $1=d-1$ iff $d=2$ ). 
3. Case 3 in our construction of points within a cone (see Figure 2) provides a substitute for the use of Pommerenke's result in [9].

Analysing the proof by Mozolyako and Havin, we are able to show the following theorem, which was conjectured by Peter W. Jones in 2003 during conversations with the first named author.

Theorem 2. Let $u$ be a positive harmonic function on the unit ball of $\mathbb{R}^{d}$ and $p$ the Poisson kernel. Then there is a point $\theta$ on the unit sphere $S$ such that

$$
\int_{B^{d}}|\nabla u(w)| p(w, \theta) d A(w)<c u(0)
$$

where $c=c\left(B^{d}\right)$ is a constant only depending on the dimension.

Note that the area or for $d \geq 3$ the volume integral is obviously larger than the line integrals defining the usual radial variation. Theorem 2 is proved in Section 4.

In Section 5 we further investigate the significance of the integral in Theorem 2. Utilizing its conformal invariance we are able to transfer it to arbitrary simply connected domains and also provide its stochastic interpretation using Brownian motion. We complement our work in Section 5 by presenting several connected open problems.

\section{Preliminaries}

We use the notation $B$ for the unit ball of $\mathbb{R}^{d}, S$ for its boundary the unit sphere, $\mathbb{D}$ for the unit disc of $\mathbb{C}, B_{r}$ or $B(r)$ for the ball with center 0 and radius $r$. The Euclidean distance between two points or a set and a point will be denoted by $d(\cdot, \cdot)$ and the diameter of a set $A$ with $\operatorname{diam}(A)$. For domains $E$ their boundary is denoted by $\partial E$, the inward unit vector of a point $x$ of $\partial E$, if it is well-defined, by $N(x)$.

Hyperbolic distance/metric On $\mathbb{D}$ the hyperbolic length of a smooth curve $\gamma$ is given by

$$
2 \int_{\gamma} \frac{d|z|}{1-|z|^{2}}
$$

The hyperbolic distance between two points $z_{0}$ and $z_{1}$ is the infimum of the hyperbolic lengths of all piecewise smooth curves in $\mathbb{D}$ with endpoints $z_{0}$ and $z_{1}$. It is invariant under conformal self-mappings of the disc. The geodesics in this metric are circles orthogonal to $\{|z|=1\}$. The distance from 0 to an arbitrary point $z_{0} \in \mathbb{D}$ is given by $\log \left(\frac{1+\left|z_{0}\right|}{1-\left|z_{0}\right|}\right)$. See [15, Section 4.6]. 
Bloch functions A function $b$ on the unit ball $B$ of $\mathbb{R}^{d}$ is called a Bloch function if it is harmonic and the semi-norm $\|b\|:=\sup _{z \in B}|\nabla b(z)|(1-|z|)$ is finite. Bloch functions are Lipschitz with respect to the hyperbolic metric. This means by definition that there is a constant $L$ such that for all $z, w \in B^{d}$

$$
|b(z)-b(w)| \leq L d_{h}(z, w)
$$

where $d_{h}(z, w)$ is the hyperbolic distance between $z$ and $w$. See [15, Section 4.2].

Poisson kernel The Poisson kernel on the unit ball $p: \mathbb{B} \times S \rightarrow \mathbb{R}$ is given by $p(z, \zeta):=\frac{1-|z|^{2}}{\omega_{d-1}|\zeta-z|^{d}}$ for $|z|<1,|\zeta|=1$ and $\omega_{d-1}$ the surface area of the unit sphere. Especially in Section 4 we use $p_{r}(\theta, \zeta)$ instead of $p(r \theta, \zeta)$, generating a family of kernels $\left(p_{r}\right)_{r \in[0,1)}$. Analogously, we write $u_{r}(\zeta)$ for $u(r \zeta)$ for functions $u$ on the unit ball.

Green's function A Green's function for a domain $\Omega$ is a function $g: \Omega \times \Omega \rightarrow$ $(-\infty, \infty]$ such that for each $w \in \Omega$

1. $g(\cdot, w)$ is harmonic on $\Omega \backslash\{w\}$ and bounded outside each neighbourhood of $w$

2. $g(w, w)=\infty$ and as $z \rightarrow w$

$$
g(z, w)= \begin{cases}\log |z|+O(1) & w=\infty \\ -\log |z-w|+O(1) & w \neq \infty\end{cases}
$$

3. $g(z, w) \rightarrow 0$ as $z \rightarrow \zeta$ and $\zeta \in \partial \Omega$.

See [16, Section 4.4].

Harnack's inequality We will use Harnack's inequality to compare values of positive harmonic functions and to get a bound for their gradients.

Theorem 3. Let $h$ be a positive harmonic function on the disc $B(w, \rho)$. Then for $r<\rho$ and $0 \leq t<2 \pi$

$$
\frac{\rho-r}{\rho+r} \leq h\left(w+r e^{i t}\right) \leq \frac{\rho+r}{\rho-r} .
$$

See [16, Theorem 1.3.1].

Harmonic measure and harmonic majorant We use the notation $w^{z_{0}}(F, E)$ for the harmonic measure with pole $z_{0}$ of $F \subset \partial E$. A harmonic majorant of a function on a given domain is a harmonic function which is pointwise larger or equal to the function. 
Martin boundary To define the Martin boundary of a domain we consider $M(x, y):=\frac{g(x, y)}{g\left(x_{0}, y\right)}$ where $x_{0}$ is a fixed point in the domain. The function $x \mapsto M(x, y)$ is continuous for $y \in \Omega \backslash\{x\}$. We now use the theorem of Constantinescu-Cornea (see $[1$, Theorem 7.2$]$ or $\left[5\right.$, Theorem 12.1]) to get a compact set $\Omega^{*}$, unique up to homeomorphisms such that

1. $\Omega$ is a dense subset of $\Omega^{*}$,

2. for each $y \in \Omega$ the function $x \mapsto M(x, y)$ has a continuous extension to $\Omega^{*}$ and

3. the extended functions separate points of $\Omega^{*} \backslash \Omega$.

The set $\Omega^{*} \backslash \Omega$ is the Martin boundary of $\Omega$ and denoted by $\partial_{M} \Omega$. The extensions of $M$ are called Martin kernels and denoted by $k^{\Omega}$. Martin kernels provide the following fundamental representation theorem for positive harmonic functions.

Theorem 4. For every positive harmonic function $h$ on $\Omega$ there is a measure $\nu$ concentrated on $\partial_{M} \Omega$ such that

$$
h(x)=\int k^{\Omega}(x, y) d \nu(y)
$$

See [1, Section II.7] or [5, Chapter 12].

Riemann mapping theorem In the last section we will use the Riemann mapping theorem to transfer Theorem 2 to arbitrary simply connected domains.

Theorem 5. Let $\Omega$ be a simply connected proper subdomain of $\mathbb{C}$ and $w_{0} \in \Omega$. Then there is a conformal map $\gamma: \Omega \rightarrow \mathbb{D}$ with $\gamma\left(w_{0}\right)=0$.

See [16, Theorem 4.4.11].

Prime ends In a simply connected and bounded domain $\Omega \subset \mathbb{C}$ a crosscut $C$ of $\Omega$ is an open Jordan arc in $\Omega$ such that $\bar{C}=C \cup\{a, b\}$ with $a, b \in \partial \Omega$. A sequence $\left(C_{n}\right)$ of crosscuts of $\Omega$ is called a null-chain if $\bar{C}_{n} \cap \bar{C}_{n+1}=\varnothing, \operatorname{diam}\left(C_{n}\right) \rightarrow 0$ and $C_{n+1}$ and $C_{0}$ are in different components of $\Omega \backslash C_{n}$. The component of $\Omega \backslash C_{n}$ not containing $C_{0}$ is called $V_{n}$. Two null-chains $\left(C_{n}\right)$ and $\left(C_{n}^{\prime}\right)$ are equivalent if for every sufficiently large $m$ there exists $n$ such that $V_{n} \subset V_{m}^{\prime}$ and $V_{n}^{\prime} \subset V_{m}$. The equivalence classes of null-chains are called prime ends of $\Omega$. The set of prime ends is denoted by $P(\Omega)$.

The ordinary topology on $\Omega$ is extended in the following way. For a subdomain $A \subset \Omega, \mathcal{E}_{A}$ is the set of prime ends that contain a null-chain whose crosscuts all lie in $A$. We define the new topology by adding the set $A \cup \mathcal{E}_{A}$ as a neighbourhood of each point in $A$ and each prime end in $\mathcal{E}_{A}$. In this extended topology $\Omega$ is dense in $\Omega \cup P(\Omega), \Omega \cup P(\Omega)$ is a compact space and therefore called prime end compactification of $\Omega$. Now the following holds true. 
Theorem 6. If $\gamma: \Omega \rightarrow \mathbb{D}$ is a conformal homeomorphism, it can be extended to a homeomorphism $\widehat{\gamma}$ between the prime end compactification of $\Omega$ and $\overline{\mathbb{D}}$.

The prime ends satisfy the conditions (1-3) of the Martin boundary. As the Martin boundary is unique up to homeomorphisms, for simply connected domains prime ends and Martin boundary coincide in this sense. See [15, Section 2.4] or [3, Chapter 9].

Coarea formula For an open set $\Omega \subset \mathbb{R}^{d}$ a real-valued Lipschitz function $a$ and an $\mathcal{L}^{1}$ function $b$ we have

$$
\int_{\Omega}|\nabla a(x)| b(x) d x=\int_{\mathbb{R}} \int_{a^{-1}(t)} g(x) d \sigma_{t}(x) d t
$$

where $\sigma_{t}$ is the $n-1$ dimensional Hausdorff-measure on the preimage of $t$ under the function $a$. See [4, Section 3.4.2].

Brownian motion and local time We call $\left(X_{t}\right)_{t \in \mathbb{R}^{+}}$Brownian motion started at 0 if it is a stochastic process on a probability space $(\Omega, \mathcal{F}, \mathbb{P})$ such that

1. $X_{0}=0 \mathbb{P}$-almost surely,

2. the increments $X_{t_{1}}-X_{t_{0}}, X_{t_{2}}-X_{t_{1}}, X_{t_{m}}-X_{t_{m-1}}$ are independent for $0 \leq$ $t_{1}<t_{2}<\ldots<t_{m}$

3. $X_{t}-X_{s} \sim \mathcal{N}(0, t-s)$ for $0 \leq s \leq t, \quad$ in other words $\mathbb{P}\left(X_{t}-X_{s} \in A\right)=$ $\frac{1}{\sqrt{2 \pi(t-s)}} \int_{A} e^{-\frac{1}{2}\left(\frac{t}{\sqrt{t-s}}\right)^{2}} d t$ and

4. the paths are almost surely continuous.

If $X^{(1)}, X^{(2)}, \ldots, X^{(d)}$ are independent Brownian motions starting in 0, the stochastic process given by $Z=\left(X^{(1)}, X^{(2)}, \ldots, X^{(d)}\right)_{t}$ is called a $d$-dimensional Brownian motion. The limit $L_{t}^{x}:=\lim _{\varepsilon \rightarrow 0} \frac{1}{2 \varepsilon} \int 1_{\left\{x-\varepsilon<X_{s}<x+\varepsilon\right\}} d s$ exists and is called local time of Brownian motion. See [1, Section I.2, I.6].

\section{Proof of Theorem 1}

The proof of Theorem 1 is based on a result of Mozolyako and Havin ([11]). We use their result to find a point in a given subset of the boundary of a Lipschitz domain such that the variation along the normal to the boundary is bounded.

The theorem of Mozolyako and Havin ([11]) will be used as stated in Theorem 7. Note however that the original statement in [11] involves $\mathcal{C}^{2}$ domains. However, it turns out - and is known - that their argument may be modified so as to work for Lipschitz domains. For the convenience of the reader we recorded in [12] a version of the Mozolyako-Havin proof, specifically taylored to Lipschitz-domains. 
Theorem 7. Let $u$ be a positive harmonic function on a Lipschitz domain $O$ with starcenter $z_{0}$ and boundary $D$. Let $N(p)$ be any direction at $p$ pointing "wellinside" the domain and $r$ be a positive function on $D$ such that $[p, p+r(p) N(p)] \subset O$ for all $p \in D$. Then for all surface balls $E \subset D$ with $\omega^{z_{0}}(E, O) \geq c$ there is a $p_{0} \in E$ and a harmonic majorant $H$ of the gradient such that

$$
\int_{0}^{r\left(p_{0}\right)} H(p+y N(p)) d y<c_{1} u\left(z_{0}\right)
$$

where the constant $c_{1}$ only depends on the Lipschitz constant of the domain, the constant $c$ and the Harnack distance between $z_{0}$ and $p_{0}+r\left(p_{0}\right) N\left(p_{0}\right)$.

Proof of Theorem 1. The proof consists of 4 parts.

1. First of all we construct Lipschitz domains on which we can use Theorem 7.

2. In the second part we look for points within a cone such that the variation along the segments connecting the points is suitably bounded.

3. Part 3 is dedicated to shifting the points constructed in part 2 onto one radius of the unit ball.

4. In the last part we collect the information of the previous parts to prove the theorem.

Construction of Lipschitz domains We want to construct a Lipschitz domain $W\left(z_{0}\right)$ for an arbitrary point $z_{0} \in B$ on which $b$ is bounded from below. In the following $M \in \mathbb{N}$ is a large enough constant only depending on $\sup _{z \in B}|\nabla b(z)|(1-|z|)$. The Lipschitz domain should satisfy the following conditions.

1 . The function $b-b\left(z_{0}\right)+M$ is positive on the whole domain.

2. The domain $W\left(z_{0}\right)$ has starcenter $z_{0}$ and the Lipschitz constant is independent of $z_{0}$.

- For $z \in B$ with $|z|>\frac{15}{16}$ we use the following notations:

$$
\begin{aligned}
I(z) & :=\{\zeta \in S:|z-\zeta| \leq 8(1-|z|)\} \\
r(z) & :=2|z|-1 \\
T(z) & :=\{w \in \mathbb{B}:|w|=r(z),|w-z| \leq 2(1-|z|)\} .
\end{aligned}
$$

The domain $V(z)$ is the convex hull of $T(z)$ and $I(z)$, intersected with $B \backslash B_{r(z)}$ (see Figure 1). 


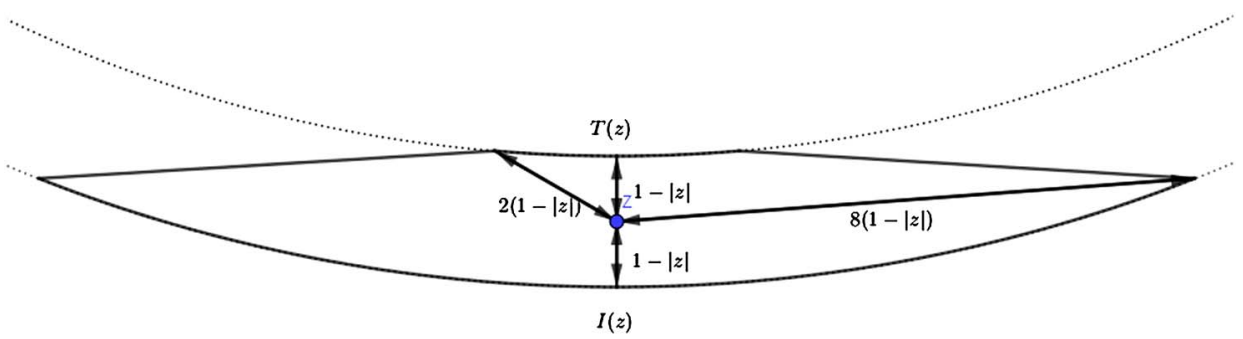

Figure 1. The domain $V(z)$.

- We consider the Whitney decomposition $\left(C_{n}\right)_{n \in \mathbb{N}}$ of $B$, so we have a decomposition of $B$ into disjoint cubes with

$$
\operatorname{diam}\left(C_{n}\right) \leq d\left(C_{n}, S\right) \leq 4 \operatorname{diam}\left(C_{n}\right)
$$

for $n \in \mathbb{N}$. Next, we fix $n \in \mathbb{N}$, check if there is a point $z \in C_{n}$ satisfying

$$
b(z)-b\left(z_{0}\right) \leq-M
$$

and set $N_{T}:=\left\{n \in \mathbb{N}: \exists z \in C_{n}: b\left(z_{n}\right)-b\left(z_{0}\right) \leq-M\right\}$. For $n \in N_{T}$ select any $z_{n} \in C_{n}$ such that $b\left(z_{n}\right)-b\left(z_{0}\right) \leq-M$ holds true. Finally, we define $T:=\left\{z_{n}: n \in N_{T}\right\}$.

- For all $z \in T$ we set $\widetilde{V}(z)=V(z)$ if $\operatorname{dist}\left(T(z), \partial V\left(z_{0}\right)\right)>\frac{1}{2}(1-|z|)$. If the condition does not hold true, we set $\widetilde{T}(z):=\{w \in \mathbb{B}:|w|=r(z),|w-z| \leq 3(1-|z|)\}$ and $\widetilde{V}(z)=\operatorname{conv}(\widetilde{T}(z) \cup I(z)) \cap\left(B \backslash B_{r(z)}\right)$. The boundary of $\widetilde{V}(z)$ consists of three disjoint sets: the top $\widetilde{T}(z)$, the bottom $\partial \widetilde{V}(z) \cap S$ and $\tilde{L}(z)$.

- The Lipschitz domain we were looking for is now given by

$$
W\left(z_{0}\right):=V\left(z_{0}\right) \backslash \bigcup_{z_{n} \in T} \tilde{V}\left(z_{n}\right)
$$

Once again the boundary of $W\left(z_{0}\right)$ consists of different parts. First, we have $T\left(z_{0}\right)$ and $\partial W\left(z_{0}\right) \cap L\left(z_{0}\right)$. The new bottom is divided into three subsets $\partial W\left(z_{0}\right) \cap S$, the tops $\partial W\left(z_{0}\right) \cap \bigcup_{z \in T} T(z)$ and $\partial W\left(z_{0}\right) \cap \bigcup_{z \in T} L(z)$.

On the tops, so for $z \in \partial W\left(z_{0}\right) \cap \bigcup_{z \in T} T(z)$ we have

$$
-2 M<b(z)-b\left(z_{0}\right)<-\frac{M}{2} .
$$

Construction of points within a cone In the following we construct a sequence of points such that the integral of a harmonic majorant of the gradient along the segments connecting two consecutive points (i.e. the variation) is bounded.

In the first step we look at $x_{0}=0$ and the corresponding domain $W(0)$. Now, by Theorem 7 , we find a point $x_{1}$ in $\partial W(0)$ such that the variation of $b$ along the line 
segment connecting $x_{1}$ and $y_{1}:=x_{1}+N\left(x_{1}\right)$ is bounded by $c_{1} M$. As $y_{1}$ has small hyperbolic distance from $x_{0}$, the variation along the interval $\left[y_{1}, x_{0}\right]$ is bounded because of Harnack's inequality.

Now we assume that points $x_{0}, \ldots, x_{n}$ and $y_{1}, \ldots, y_{n}$ are already chosen. We consider the domain $W\left(x_{n}\right)$ and scale it by a homothetic transformation such that the diameter is 100 . As $b-b\left(x_{n}\right)+M$ is a positive harmonic function on $W\left(x_{n}\right)$ we can apply Theorem 7 . We get a point $x$ on the lower part of the boundary of $W\left(x_{n}\right)$ such that the variation along the line segment connecting $x$ and $y_{n+1}:=x+N(x)$ is bounded by $c_{1} M$. We distinguish three cases.

1. If $x$ is in the unit sphere, we take $x_{n+1}:=x$ and stop the construction.

2. If $x$ is on a top, so in the set $\partial W\left(x_{n}\right) \cap \bigcup_{z \in T} T(z)$, we define $x_{n+1}:=x$.

3. If $x$ is in $\partial W\left(x_{n}\right) \cap \bigcup_{z \in T} L(z)$, we take the intersection point $y$ of the normal to the boundary at $x$ and the sphere with radius $r\left(z_{n}\right)$, which is the radius of the corresponding top, and set $x_{n+1}:=y$ (see Figure 2).

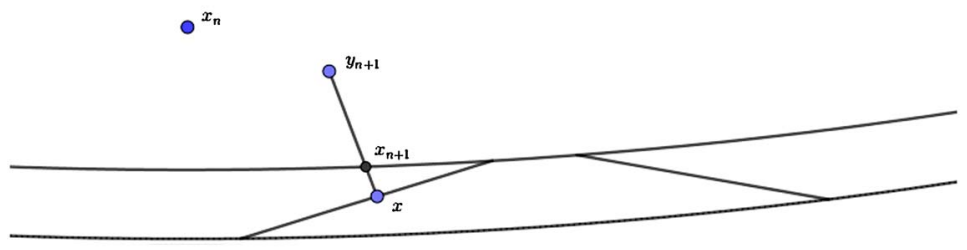

Figure 2. The case $x \in \partial W\left(x_{n}\right) \cap \bigcup_{z \in T} L(z)$.

We have constructed two sequences of points $\left(x_{n}\right)_{n \in \mathbb{N} \cup\{0\}}$ and $\left(y_{n}\right)_{n \in \mathbb{N}}$, both converging to a point on the boundary $x$. They are contained in the cone with apex $x$ and opening angle $\pi / 2$ and satisfy the following conditions:

1. $\int_{\left[y_{n+1}, x_{n+1}\right]} H_{n+1}(\zeta) d|\zeta| \leq c_{1} M$,

2. $\int_{\left[x_{n}, y_{n+1}\right]} H_{n+1}(\zeta) d|\zeta| \leq c_{1} M$

3. $b\left(x_{n}\right)-b(0) \leq-n \frac{M}{2}$ and

4. for $\zeta \in\left[x_{n}, y_{n+1}\right] \cup\left[y_{n+1}, x_{n+1}\right]$ we have $b(\zeta)-b(0) \leq-n \frac{M}{2}+2 c_{1} M$

where $H_{n+1}$ is a harmonic majorant of $|\nabla b|$ on the domain $W\left(x_{n}\right)$ (see Figure 3 ). 


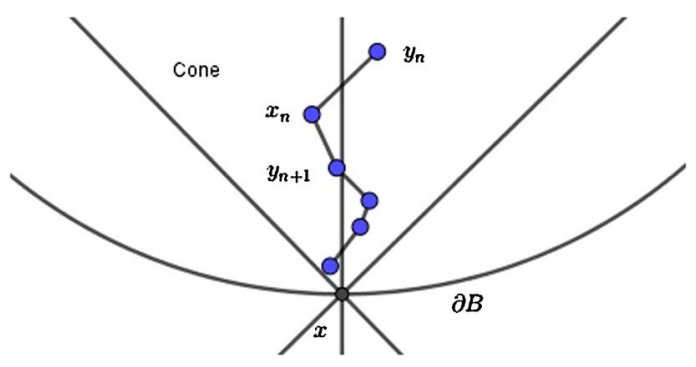

Figure 3. Cone, radius and some points.

Shifting of points and corresponding segments We want to shift the points $x_{n}$ and $y_{n}$ and the corresponding segments to the radius connecting 0 and the limit $x$ of our point sequences.

Shifting $\left[x_{n}, y_{n+1}\right]$ : As in the construction for the segment $\left[x_{n}, y_{n+1}\right]$, we can use Harnacks inequality to bound the integral of the harmonic function along the segment $\left[a_{n}, b_{n+1}\right]=\left[\left|x_{n}\right| x,\left|y_{n+1}\right| x\right]$.

Shifting $\left[y_{n+1}, x_{n+1}\right]$ : We shift $y_{n+1}$ to $b_{n+1}=\left|y_{n+1}\right| x$ and $x_{n+1}$ to $a_{n+1}=$ $\left|x_{n+1}\right| x$ and distinguish the following two cases.

Case 1: $\left|x_{n+1}\right|=1$. In this case we have already reached the unit sphere and $x_{n+1}=x$ so we already have a suitable bound for

$$
\int_{\left[b_{n+1}, a_{n+1}\right]}|\nabla b(\zeta)| d|\zeta|
$$

as $y_{n+1}=b_{n+1}$ and $x_{n+1}=a_{n+1}$.

Case 2: If $\left|x_{n+1}\right| \neq 1$, we note that the distance of $x_{n+1}$ to the radius is bounded by $c\left|x_{n+1}\right|$ because the point is in the cone. Next, we take $s:=\left[y_{n+1}, x_{n+1}\right] \cap B(0,1-$ $\left.\frac{3}{2}\left(1-\left|x_{n+1}\right|\right)\right)$. The distance of $s$ to the boundary of $W\left(x_{n}\right)$ is at least $\frac{1}{2}\left(1-\left|x_{n+1}\right|\right)$ and therefore comparable to the distance from the radius to which we want to shift our segment. We can use Harnack's inequality once again and get a bound of

$$
\int_{\left[\left(1-\frac{3}{2}\left(1-\left|x_{n+1}\right|\right)\right) x, b_{n+1}\right]}|\nabla b(\zeta)| d|\zeta|
$$

where $b_{n+1}:=\left|y_{n+1}\right| x$ (see Figure 4$)$.

The segment $\left[a_{n+1},\left(1-\frac{3}{2}\left(1-\left|x_{n+1}\right|\right)\right) x\right]$ with $a_{n+1}:=\left|x_{n+1}\right| x$ is left. Here we use the next harmonic majorant $H_{n+2}$ on the domain $W\left(x_{n+1}\right)$. There we can easily bound 


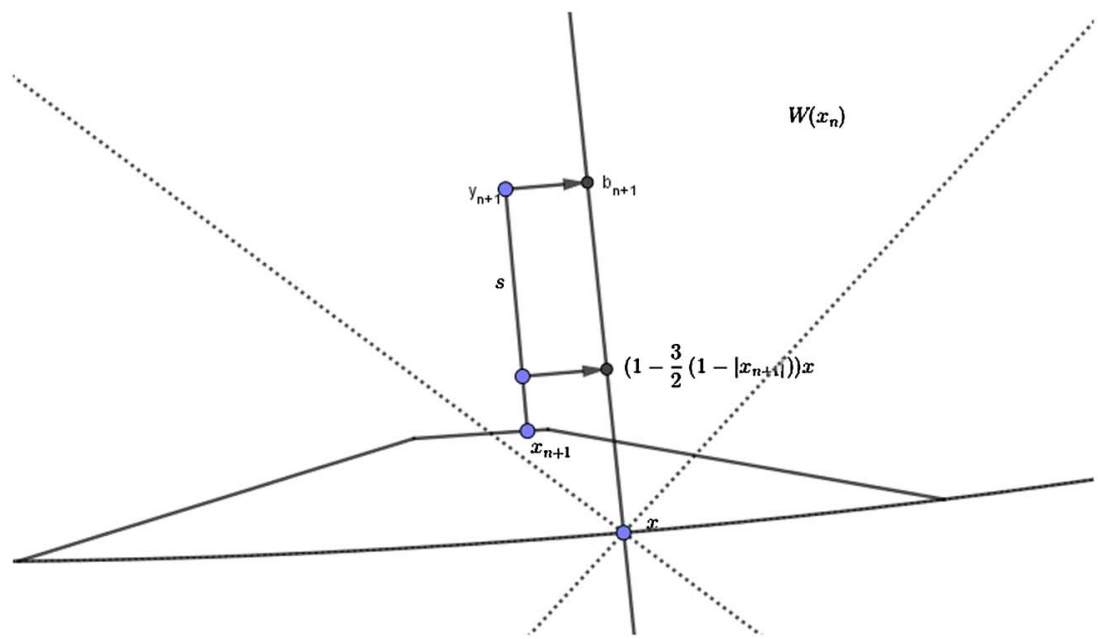

Figure 4. Case 2.

$$
\int_{\left[\left|x_{n+1}\right| x,\left(1-\frac{3}{2}\left(1-\left|x_{n+1}\right|\right)\right) x\right]} H_{n+2}(\zeta) d|\zeta|
$$

by $c_{1} M$.

Collecting the information We obtained a point $x$ on the unit sphere and sequences of points $\left(a_{n}\right)$ and $\left(b_{n}\right)$ on $[0, x]$ both converging to $x$ that satisfy:

1. $\left|a_{n}\right|<\left|b_{n+1}\right|<\left|a_{n+1}\right|$

2. $\int_{\left[b_{n+1}, a_{n+1}\right]}|\nabla b(\zeta)| d|\zeta| \leq c_{1} M$

3. $\int_{\left[a_{n}, b_{n+1}\right]}|\nabla b(\zeta)| d|\zeta| \leq c_{1} M$

4. $b\left(a_{n}\right)-b(0) \leq-n \frac{M}{2}$

5. for $\zeta \in\left[a_{n}, b_{n+1}\right] \cup\left[b_{n+1}, a_{n+1}\right]$ we have $b(\zeta)-b(0) \leq-n \frac{M}{2}+2 c_{1} M$.

To finish the proof we can write this in a more compact form as

$$
\begin{aligned}
& \int_{[0, x]}|\nabla b(\zeta)| e^{b(\zeta)} d|\zeta|= \\
& =\sum_{n=0}^{\infty} \int_{\left[a_{n+1}, b_{n+1}\right]}|\nabla b(\zeta)| e^{b(\zeta)} d|\zeta|+\int_{\left[b_{n+1}, a_{n}\right]}|\nabla b(\zeta)| e^{b(\zeta)} d|\zeta| \leq \\
& \leq 2 e^{b(0)+2 c_{1} M} \sum_{n=0}^{\infty} e^{-n \frac{M}{2}} c_{1} M<c\left(c_{1}, M, b(0)\right) .
\end{aligned}
$$




\section{Proof of Theorem 2}

In the previous section we used the result of Mozolyako and Havin ([11]) to prove Theorem 1. In this section we present a result of the analysis of the proof given in [11] to prove Theorem 2.

Let $u$ be a positive harmonic function on $B^{d}$. Following the notation of Mozolyako and Havin in [11] we define $\sigma(x)$ as the normalized gradient of $u$ at the point $x$. By differentiating the Poisson kernel with respect to the first variable in direction $\sigma(x)$, we get a new kernel $c_{r}(\zeta, \theta):=\frac{\partial p}{\partial \sigma\left(r^{2} \zeta\right)}(r \zeta, \theta)$. We define the kernel $b_{r}(\zeta, \theta):=\int p_{r}(\zeta, x) c_{r}(x, \theta) d x$ and $B_{r}$ denotes the integral operator $B_{r}(f)=$ $\int_{S} b_{r}(\zeta, \theta) f(\theta) d \theta$.

Mozolyako and Havin ([11]) proved that for every positive harmonic function $u$ on $B^{d}$ there is a probability measure $\nu$ such that

$$
J=\int_{S} \int_{0}^{1} B_{r}\left(u_{r}\right)(\zeta) d r d \nu(\zeta) \leq c u(0)
$$

Let $V(u)$ be the set of points in $S$ such that the radial variation of $u$ is bounded. In [11] they showed that for every point $p \in S$ and $\rho>0$ the Hausdorff dimension of $V(u) \cap B^{d}(p, \rho)$ is $n-1$. We use the same fact to prove Theorem 2 .

Proof. First we rewrite the integral in the theorem as follows

$$
\int_{B^{d}}|\nabla u(w)| p(w, \theta) d A(w)=\int_{S} \int_{S} \int_{0}^{1} c_{\sqrt{r}}(\zeta, \alpha) u_{\sqrt{r}}(\alpha) p_{r}(\zeta, \theta) r d r d \zeta d \alpha .
$$

Using the symmetry of $p_{r}$ and because $p_{r} \leq 2 p_{\sqrt{r}}$ pointwise, we obtain

$$
\int_{0}^{1} 2 B_{\sqrt{r}}\left(u_{\sqrt{r}}\right)(\theta) d r
$$

as an upper bound for our integral and by a simple substitution this is equal to

$$
4 \int_{0}^{1} B_{s}\left(u_{s}\right)(\theta) d s
$$

This integral is bounded by $c u(0)$ for at least one $\theta$ as $\nu$ is a probability measure. 
By similar computation

$$
I=\int_{S} \int_{S} \int_{0}^{1} P_{r}\left(\left|\nabla u_{r}(\zeta)\right|\right) p_{r}(\zeta, \theta) d r d \zeta d \nu(\theta)
$$

where $P_{r}$ is the operator with integral kernel $p_{r}$, is also bounded.

\section{Discussion and related open problems}

In the following discussion we exploit the remarkable flexibility of the integral

$$
\int_{B^{d}}|\nabla u(w)| p(w, \theta) d A(w)
$$

in Theorem 2, in particular its conformal invariance and its connection to Brownian martingales.

First application of the coarea formula By applying the coarea formula to the integral in Theorem 2 we get that

$$
\int_{0}^{\infty} \int_{u^{-1}(c)} p(w, \theta) d H_{n-1}(w) d c<c u(0)
$$

where $H_{n-1}$ is the $n-1$ dimensional Hausdorff measure and $u^{-1}(c)$ is the preimage of the value $c$. For a harmonic function $v$ with $|v|<A$ we get

$$
\int_{-A v^{-1}(c)}^{A} \int_{p(w, \theta) d H_{n-1}(w) d c}
$$

and as there is a $\theta$ such that the expression is bounded, we know that $\int_{v^{-1}(c)} p(w, \theta) d H_{n-1}$ is in $\mathcal{L}^{1}([-A, A])$. Therefore we can ask the following question:

Open Problem 1. For which $p>1$ exists a $\theta$ such that the integrand $\int_{v^{-1}(c)} p(w, \theta) d H_{n-1}$ is in $\mathcal{L}^{p}([-A, A])$ ? 
Simply connected domains In order to get from an arbitrary simply connected domain to the unit disc we use the Riemann mapping theorem.

We make use of two concepts regarding the boundary of simply connected domains. The first one is the Martin boundary of a domain (cf. [1] or [5]) and the second one is the concept of prime ends introduced by Carathéodory (cf. [15] and [3]). For simply connected domains, prime ends and the Martin boundary coincide up to homeomorphisms.

We can now take advantage of the conformal invariance and transfer our result of Theorem 2 to this setting.

Theorem 8. Let $\Omega$ be a simply connected bounded domain, $v$ a positive harmonic function on $\Omega, k^{\Omega}$ the Martin kernel and $g^{\Omega}\left(\cdot, w_{0}\right)$ the Green's function with singularity in $w_{0} \in \Omega$. Then there is a prime end $\zeta$ such that

$$
\int_{\Omega}|\nabla v(w)| k^{\Omega}(w, \zeta)\left|\nabla g^{\Omega}\left(w, w_{0}\right)\right| d A(w)<c
$$

where $c$ is a constant only depending on the value $v\left(w_{0}\right)$ and the domain $\Omega$.

Proof. Let $\gamma: \Omega \rightarrow \mathbb{D}$ be a Riemann map with $\gamma\left(w_{0}\right)=0$. The function $u:=v \circ f$, where $f$ is the inverse of $\gamma$, is a positive harmonic function on $\mathbb{D}$. So we know by Theorem 2 that there is a $\theta \in \mathbb{T}$ with

$$
\int_{\mathbb{D}}|\nabla(v \circ f)(z)| p(z, \theta) d A(z)<c v\left(w_{0}\right) .
$$

By substitution we get that

$$
\int_{\Omega}|\nabla v(w)| p(\gamma(w), \theta)|| \gamma^{\prime}(w) \mid d A(w)<c v\left(w_{0}\right) .
$$

Next we use that $p(\gamma(w), \theta)$ is the Martin kernel $k^{\Omega}(w, \zeta)$ where $\zeta$ is the prime end of $\Omega$ such that the extension $\widehat{\gamma}$ of $\gamma$ to the prime ends of $\Omega$ satisfies $\widehat{\gamma}(\zeta)=\theta$ and get

$$
\int_{\Omega}|\nabla v(w)| k^{\Omega}(w, \zeta)\left|\gamma^{\prime}(w)\right| d A(w)<c v\left(w_{0}\right) .
$$

By calculation we know $\left|\gamma^{\prime}(w)\right|=\left|\nabla g^{\Omega}\left(w, w_{0}\right)\right||\gamma(w)|$ where $g^{\Omega}$ is the Green's function of $\Omega$. Therefore we obtain

$$
\int_{\Omega}|\nabla v(w)| k^{\Omega}(w, \zeta)\left|\nabla g^{\Omega}\left(w, w_{0}\right)\right||\gamma(w)| d A(w)<c v\left(w_{0}\right) .
$$


We now use the fact that for bounded simply connected plane domains the integral of the gradient of the Green's function

$$
\int_{\Omega}\left|\nabla g^{\Omega}(w, z)\right| d A(z)
$$

is bounded by a constant only depending on $\Omega$ (see [4]). Partitioning the domain into one part where $|\gamma|<\frac{1}{2}$ and one where $|\gamma| \geq \frac{1}{2}$ leads to the following. On the domain where $|\gamma|<\frac{1}{2}$ we know that $k^{\Omega}$ and $|\nabla v|$ are bounded by constants only depending on the domain $\Omega$ and the value $v\left(w_{0}\right)$. On the second part we only use that $|\gamma| \geq \frac{1}{2}$. Then we obtain

$$
\int_{\Omega}|\nabla v(w)| k^{\Omega}(w, \zeta)\left|\nabla g^{\Omega}\left(w, w_{0}\right)\right| d A(w)<c
$$

where the constant $c$ only depends on the value $v\left(w_{0}\right)$ and $\Omega$.

The area integral in Theorem 8 depends expressly on the Green's function and the Martin kernels of the simply connected domain $\Omega \subset \mathbb{C}$ and not on the Riemann map itself. This allows us to consider it in more general domains, either in multiply connected domains or in higher dimensions.

Open Problem 2. Let $v$ be a positive harmonic function on a not necessarily simply connected domain $\Omega \subset \mathbb{C}$ with Green's function $g^{\Omega}$. Is there an element $\zeta$ of the Martin boundary of $\Omega$ such that the integral

$$
\int_{\Omega}|\nabla v(w)| k^{\Omega}(w, \zeta)\left|\nabla g^{\Omega}\left(w, w_{0}\right)\right| d A(w)
$$

is bounded? Even for Denjoy domains, that is when $\partial \Omega \subset \mathbb{R}$, this problem seems to be open.

We refer to Jerison and Kenig ([8]) for the concept of nontangentially accessible (NTA) domains and can ask the following.

Open Problem 3. Let $v$ be a positive harmonic function on a nontangentially accessible domain $\Omega \subset \mathbb{R}^{d}$. Is there an element $\zeta$ of the Martin boundary of $\Omega$ such that the integral

$$
\int_{\Omega}|\nabla v(w)| k^{\Omega}(w, \zeta)\left|\nabla g^{\Omega}\left(w, w_{0}\right)\right| d A(w)
$$

is bounded? The problem is even open for Lipschitz domains. 
Second application of the coarea formula We continue by transforming the integral

$$
\int_{\Omega}|\nabla v(w)| k^{\Omega}(w, \zeta)\left|\nabla g^{\Omega}\left(w, w_{0}\right)\right||\gamma(w)| d A(w)
$$

Using the coarea formula we get that the integral

$$
\int_{0}^{\infty} \int_{g^{-1}(c)}|\nabla v(w)| k^{\Omega}(w, \zeta)(w)|\gamma(w)| d \sigma_{c}(w) d c
$$

is bounded. In fact, we apply the coarea formula to a partition of $\Omega$ into subdomains where the Green's function is Lipschitz and put the results together. As we know that $|\gamma(w)|=e^{-g\left(w, w_{0}\right)}$, the expression

$$
\int_{0}^{\infty} \int_{g^{-1}(c)}|\nabla v(w)| k^{\Omega}(w, \zeta)(w) d \sigma_{c}(w) e^{-c} d c
$$

where $\sigma_{c}$ is the Hausdorff-measure, is bounded. As this implies that

$$
\int_{g^{-1}(c)}|\nabla v(w)| k^{\Omega}(w, \zeta)(w) d \sigma_{c}(w)
$$

is in $\mathcal{L}^{1}\left([0, \infty), e^{-c} d c\right)$, we can ask the following question.

Open Problem 4. Is there a $\zeta$ such that the function $c \mapsto$ $\int_{g^{-1}(c)}|\nabla v(w)| k^{\Omega}(w, \zeta)(w) d \sigma_{c}(w)$ is in $\mathcal{L}^{p}\left([0, \infty), e^{-c} d c\right)$ for $p>1$ ?

Brownian martingales We also want to present the link to stochastic analysis. Therefore, let $Z_{t}$ be the $d$-dimensional Brownian motion stopped at the unit sphere. We apply the formula

$$
\mathbb{E}\left(\int_{0}^{\tau} m\left(Z_{t}\right) d t\right)=\int_{B^{d}} m(z) g(z, 0) d A(z)
$$

for bounded continuous functions $m$, the Green's function $g$ and Brownian motion $Z_{t}$. Now the integral in Theorem 2 can be written as

$$
\int_{B^{d}}|\nabla u(w)| p(w, \theta) d A(w)=\mathbb{E}\left(\int_{0}^{\tau}\left|\nabla u\left(Z_{t}\right)\right| p\left(Z_{t}, \theta\right) \frac{1}{g\left(0, Z_{t}\right)} d t\right) .
$$

As $u\left(Z_{t}\right)$ can be written as $\int_{0}^{t} \nabla u\left(Z_{s}\right) d Z_{s}$ for $t<\tau$, a new question arises naturally: 
Open Problem 5. For $F: \Omega \rightarrow \mathbb{R}^{+}$with $F=\int_{0}^{\tau} G_{t} d Z_{t}+F_{0}$, where $G_{t}$ is a martingale, show that there is a $\theta$ such that $\mathbb{E}\left(\int_{0}^{\tau}\left|G_{t}\right| \frac{p\left(Z_{t}, \theta\right)}{g\left(0, Z_{t}\right)} d t\right)<c$.

Using the local time of Brownian motion $L_{\tau}^{w}$ and the fact $\mathbb{E}\left(L_{\tau}^{w}\right)=g(0, w)$ we could also rewrite the integral of Theorem 2

$$
\int_{B^{d}}|\nabla u(w)| p(w, \theta) \frac{1}{g(0, w)} \mathbb{E}\left(L_{\tau}^{w}\right) d A(w)=\mathbb{E}\left(\int_{B^{d}}|\nabla u(w)| p(w, \theta) \frac{1}{g(0, w)} L_{\tau}^{w} d A(w)\right)
$$

These expressions are all bounded for at least one $\theta$ because of their equality to the integral in Theorem 2.

Acknowledgements. This paper is part of the second named author's PhD thesis written at the Department of Analysis, Johannes Kepler University Linz. The research has been supported by the Austrian Science foundation (FWF) Pr.Nr P28352-N32.

\section{References}

1. Bass, R. F., Probabilistic Techniques in Analysis, Probability and Its Applications (New York), Springer, New York, 1995. MR1329542

2. Bourgain, J., Boundedness of variation of convolution of measures, Mat. Zametki $\mathbf{5 4}$ (1993), 24-33. 158. MR1256604

3. Collingwood, E. F. and Lohwater, A. J., The Theory of Cluster Sets, Cambridge Tracts in Mathematics and Mathematical Physics 56, Cambridge University Press, Cambridge, 1966. MR0231999

4. Evans, G. C., Note on the gradient of the Green's function, Bull. Amer. Math. Soc. 38 (1932), 879-886. MR1562536

5. Helms, L. L., Introduction to Potential Theory, Pure and Applied Mathematics XXII, Wiley-Interscience A Division of John Wiley \& Sons, New York-LondonSydney, 1969. MR0261018

6. Hunt, R. A. and Wheeden, R. L., On the boundary values of harmonic functions, Trans. Amer. Math. Soc. 132 (1968), 307-322. MR0226044

7. Hunt, R. A. and Wheeden, R. L., Positive harmonic functions on Lipschitz domains, Trans. Amer. Math. Soc. 147 (1970), 507-527. MR0274787

8. Jerison, D. S. and Kenig, C. E., Boundary behavior of harmonic functions in nontangentially accessible domains, Adv. Math. 46 (1982), 80-147. MR0676988

9. Jones, P. W. and Müller, P. F. X., Radial variation of Bloch functions, Math. Res. Lett. 4 (1997), 395-400. MR1453069

10. Jones, P. W. and Müller, P. F. X., Universal covering maps and radial variation, Geom. Funct. Anal. 9 (1999), 675-698. MR1719594 
11. Mozolyako, P. A. and Havin, V. P., Boundedness of variation of a positive harmonic function along the normals to the boundary, Algebra $i$ Analiz 28 (2016), 67110. MR3604290

12. Müller, P. F. X. and Riegler, K., Radial variation of positive harmonic functions on Lipschitz domains. arXiv : 2003.07176.

13. Nicolau, A., Radial behaviour of harmonic Bloch functions and their area function, Indiana Univ. Math. J. 48 (1999), 1213-1236. MR1757073

14. O'NeILl, M. D., Vertical variation of harmonic functions in upper half-spaces, Colloq. Math. 87 (2001), 1-12. MR1812141

15. Pommerenke, C., Boundary Behaviour of Conformal Maps, Grundlehren der Mathematischen Wissenschaften [Fundamental Principles of Mathematical Sciences] 299, Springer, Berlin, 1992. MR1217706

16. Ransford, T., Potential Theory in the Complex Plane, London Mathematical Society Student Texts 28, Cambridge University Press, Cambridge, 1995. MR1334766

Paul F. X. Müller

Institute of Analysis

Johannes Kepler University Linz

Altenberger Strasse 69

A-4040 Linz

Austria

Paul.Mueller@jku.at

Received January 14, 2019

in revised form November 21, 2019
Katharina Riegler

Institute of Analysis

Johannes Kepler University Linz

Altenberger Strasse 69

A-4040 Linz

Austria

Katharina.Riegler@jku.at 